\title{
Functional characterization of Bombyx mori nucleopolyhedrovirus mutant lacking late expression factor 9
}

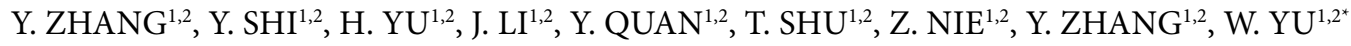 \\ ${ }^{1}$ Institute of Biochemistry, College of life sciences, Zhejiang Sci-Tech University, Zhejiang Province, Hangzhou 310018, P. R China; \\ ${ }^{2}$ Zhejiang Provincial Key Laboratory of Silkworm Bioreactor and Biomedicine, Zhejiang Province, Hangzhou 310018, P. R. China
}

Received November 18, 2015 revised February 16, 2016; accepted July 20, 2016

\begin{abstract}
Summary. - Baculoviridae is a family of invertebrate viruses with large double-stranded DNA genomes. Proteins encoded by some late expression factor (lef) genes are involved in the regulation of viral gene expression. Lef-9 is one of four transcription-specific Lefs, which are components of the virus-encoded RNA polymerase, and can initiate and transcribe late and very late genes. As a multifunctional protein encoded by the Bombyx mori nucleopolyhedrovirus (BmNPV), Lef-9 may be involved in the regulation of viral propagation. However, the underlying mechanism remains unclear. To determine the role of lef-9 in baculovirus infection, lef-9-knockout virus (lef-9-KO-Bacmid virus) was constructed using the Red recombination system, and the Bac-to-Bac system was used to prepare lef-9-repaired virus (lef-9-Re-Bacmid virus). The lef-9-KO virus did not produce infectious viruses or show infection activity, while the lef-9-repaired virus recovered both. Quantitative real-time polymerase chain reaction (qRT-PCR) analysis of the transcription levels in wild-type-Bacmid, lef9-KO-Bacmid, and lef-9-Re-Bacmid viruses showed that the lef-9-KO bacmid had little effect on viral genome replication. However, the transcription levels of the early and late viral genes, lef-3, ie-1, vp39, and $p 10$, were significantly lower in $\mathrm{BmN}$ cells transfected with lef-9-KO-Bacmids than in the controls. Electron microscopy showed no visible enveloped virions in cells transfected with lef-9-KO-Bacmids, while many mature virions in cells transfected with lef-9-Re-Bacmid and wt-Bacmid were present. Thus, lef-9 was not essential for viral genome replication, but significantly affected viral gene transcription and expression in all periods of cell life cycle.
\end{abstract}

Keywords: Bombyx mori nucleopolyhedrovirus; lef-9 gene; virus replication; virus transcription; virus assembly

\section{Introduction}

Baculoviridae is a family of viruses with large doublestranded DNA genomes. To date, more than 600 baculoviruses have been described (Herniou and Jehle, 2007; Yin et al., 2015), and the genomes of 47 types of baculoviruses have been sequenced. The sizes of these genomes are between $80-180$ $\mathrm{kb}$, with $90 \%$ of genes involved in protein coding (Ayres et al.,

*Corresponding author. E-mail: mikkyu@163.com; phone: +86571-86843190.

Abbreviations: $\mathrm{BmNPV}=$ Bombyx mori nucleopolyhedrosis virus; $\mathrm{BmN}=$ Bombyx mori cells; $\mathrm{BV}=$ budding virus; $\mathrm{Lef}=$ late expression factor
1994). The Bombyx mori nucleopolyhedrovirus (BmNPV) is a model baculovirus. Its genome structure is very similar to the Autographa californica multiple NPV (AcMNPV) genome. AcMNPV is the first completely sequenced and well-studied baculovirus (Ayres et al., 1994; Bulach et al., 1999; Herniou et al., 2003; Jehle et al., 2006). The genome of this virus contains $133,894 \mathrm{bp}$, with an average A-T content of 59\%, and 154 open reading frames (ORFs). In 1999, the BmNPV (T3 strain) genome was identified to contain 128,413 bp and 135 ORFs encoding 60 or more amino-acid residues (Gomi et al., 1999). Comparison of the ORFs in the genomes of the two viruses revealed that 115 ORFs were highly conserved, with more than 90\% homology (Yu et al., 2013).

Proteins called late expression factors (LEFs) are transcribed and translated by 19 viral late genes identified by 
transient expression assays (Hang et al., 1995; Rapp et al., 1998). Among the 19 genes, p143, ie-2, dnapol, lef-2, ie-1, $p 35$, lef-1, lef-3, and lef-7 were found to influence viral DNA replication (Iyengar et al., 1999; Vanarsdall et al., 2006), while lef-4, lef-5, lef-6, lef-8, lef-9, lef-10, lef-12, $p 47$, and $p p 31$ were found to act as late transcription genes (Lu and Miller, 1995). The four subunits of lef-4, lef-8, lef-9, and $p 47$ (Guarino et al., 1998) were found to encode viral RNA polymerase (Vanarsdall et al., 2005), while lef-5, lef-6, lef-10, lef-12, and $p p 31$ were found to be directly involved in the regulation of late gene transcription (Lu and Miller, 1995). Late genes are expressed during viral DNA replication and are soon afterwards regulated by RNA polymerase.

The lef-9 gene is a highly conserved gene in insect baculoviruses (Rose et al., 2013). The site of lef-9 in the BmNPV genome is at the position 44,402-45,874 with length of 1473 bp. The gene encodes 491 amino acids, and the predicted molecular mass of the Lef- 9 protein is $54 \mathrm{~K}$. The predicted amino acid sequences of $l e f-9$ contain motifs that are similar to those that participate in the catalytic centers of known RNA polymerases (Crouch et al., 2007). Although one study has shown that lef-9 is a subunit of RNA polymerase (Guarino et al., 1998), which regulates the transcription of late and very late genes. Several reports have shown that lef- 9 takes part in many other functions. $\mathrm{Wu}$ et al. found

Table 1. Primer sequences for PCR amplification

\begin{tabular}{|c|c|}
\hline Primers & Primer sequences \\
\hline lef-9-CF* & $\begin{array}{l}\text { Forward: 5'-TGTTTTCTTTTTTGGATAAAACT } \\
\text { CCTACTGAGTT } \\
\text { TGACCTCATAT TAGACGTGTAGGCTGGAGCTGC } \\
\text { TTC-3' }\end{array}$ \\
\hline lef-9-CR* & $\begin{array}{l}\text { Reverse:5'-TGATAAAATTTTTTAAAATCACTTTAAA } \\
\text { TTCTTCA } \\
\text { T T GGTA A A A A AT GCC AT G G G A AT TA GCC A } \\
\text { TGGTCC-3' }\end{array}$ \\
\hline lef-9F & Forward: 5'-CACGTTTTCGTCGATTTG-3' \\
\hline lef-9R & Reverse: 5'-CGGCAAATCCGTGACTAT-3' \\
\hline cat $\mathrm{F}$ & Forward: 5'-CACGTTTAAATCAAAACTGGTG-3' \\
\hline cat $\mathrm{R}$ & Reverse: 5'-CAATATGGACAACTTCTTCG-3' \\
\hline pFblef-9F & $\begin{array}{l}\text { Forward: 5'-CGGGATCCTCAGCCTGTTGTCG } \\
\text { TGAATACC-3' }\end{array}$ \\
\hline pFblef-9R & $\begin{array}{l}\text { Reverse: 5'-CGGAATTCCGTATTGAGATTTTCC } \\
\text { GCAGTT-3' }\end{array}$ \\
\hline$g p 41 \mathrm{~F}$ & Forward: 5'-CGTAGTGGTAGTAATCGCCGC-3' \\
\hline$g p 41 \mathrm{R}$ & Reverse: 5'-AGTCGAGTCGCGTCGCTTT-3' \\
\hline$\beta$-actin $\mathrm{F}$ & Forward: 5'-GCGCGGCTACTCGTTCACTACC-3' \\
\hline$\beta$-actin $\mathrm{R}$ & Reverse: 5'-TGCCGCAAGCTTCCATACCC-3' \\
\hline M13F & Forward: 5'-GTTTTCCCAGTCACGAC-3' \\
\hline M13R & Reverse: 5'-CAGGAAACAGCTATGAC-3' \\
\hline
\end{tabular}

*These two primers were composed of 50-bp lef-9 homology arms (underlined) and a 20-bp cat homologous zone (underlined). Boxed sequences represent the restriction enzymes BamHI and EcoRI. that it could interact with IE-2 in silkworm cells to regulate viral gene expression and viral replication (Wu et al., 2013). These results implied that $l e f-9$ might have multiple functions involved in the regulation of viral replication, but the details of the process and mechanism of viral gene replication and transcription are still not clear.

Many experimental studies have shown that gene knockout technology is a powerful tool for determining gene function. It has also been used to study baculovirus genes (Su et al., 2011; Lin and Blissard, 2002; Shi et al., 2015). In this study, lef-9-KO bacmids were constructed using gene knockout technology with a Red recombination system, and were used to study the effect of this defect virus on infectious virus production, and viral replication and transcription, in comparison with wild-type virus (BmNPV) and lef-9repaired virus.

\section{Materials and Methods}

Viruses and cells. Bombyx mori cells (BmN, preserved in our laboratory) were cultured at $27^{\circ} \mathrm{C}$ in Sf-900 medium (Thermo Fisher Scientific, USA) supplemented with 10\% fetal bovine serum. Virus (Bombyx mori nucleopolyhedrosis virus, BmNPV) was preserved in our laboratory.

Construction of lef-9-KO and lef-9-repaired bacmids. To construct the lef-9-KO bacmid, we PCR-amplified linearized lef- 9 gene fragments. The $1100 \mathrm{bp}$ long target fragment, named lef-9-C, was amplified by using the plasmid pKD3 (encoding a chloramphenicol acetyltransferase [cat] gene) (Youbio, China) as a template, and lef-9-CF and lef-9-CR as primers (Table 1). The lef-9-C fragment was transformed into DH10Bac competent cells (Takara, Japan) containing the plasmid pKD46 (Youbio, China) for the expression of homologous recombination enzymes required for the Red recombination system (Copeland et al., 2001; Costantino and Court, 2003), and induced by $L$-Arabinose (Promega, USA). Bacteria were cultured for $24 \mathrm{hr}$ at $37^{\circ} \mathrm{C}$ on agar plates with chloramphenicol and kanamycin. The positive colonies were selected and confirmed by PCR using the following primer pairs: lef-9F and cat $\mathrm{R}$, lef- $9 \mathrm{R}$ and cat $\mathrm{F}$, and $l e f-9 \mathrm{~F}$ and lef-9R (Table 1).

To construct repaired bacmids (lef-9-Re-Bacmid), the lef-9 gene was cloned into the vector pFastBacHTB (Tiandz Inc., China) and then inserted downstream of the polyhedrin promoter by using the Bac-to-Bac system (preserved in our laboratory). A fragment containing the lef-9 gene with its native promoter was amplified by PCR using the primers pFblef-9F and pFblef-9R (Table 1). The repaired fragment was cloned into pFastBacHTB to obtain recombinant plasmid pFastBacHTB-lef-9, which was transformed into DH10Bac competent cells containing the lef-9-KO bacmid prepared previously. Plates containing kanamycin, 5-bromo- 
4-chloro-3-indolyl- $\beta$-d-galactopyranoside (X-gal), gentamicin, tetracycline, and isopropyl $\beta$-d-1-thiogalactoside (IPTG) were used to screen positive colonies. The primer combinations M13F and M13R, M13F and pFblef-9F, and M13R and pFblef-9R and pFblef-9F/R (Table 1) were used to confirm positive colonies. The construction map is shown in Fig. 1.

Real-time quantitative PCR assay. To analyze the effects of the lef- 9 gene on viral DNA replication, we transfected lef-9-KO-Bacmid, wt-Bacmid (wild type), and lef-9-ReBacmid DNA $(1 \mu \mathrm{g}$ each $)$ into BmN cells $\left(1 \times 10^{6} / 35-\mathrm{mm}\right.$ dish), and collected the transfected cells after $6,12,18,24$, and $48 \mathrm{hr}$. We then analyzed the transcription level of the baculovirus $g p 41$ gene in the infected cells by performing quantitative real-time PCR (qRT-PCR) at various time points, and the viral $g p 41$ gene-specific primers gp $41 \mathrm{~F}$ and gp41R were used (Table 1). $\beta$-Actin was used as an internal control. To analyze early and late viral gene transcription at different transcription times after cell transfection, we used $i e-1$ and lef-3 as early gene controls (Kejju et al., 1999). The $v p 39$ and $p 10$ genes were used as late and very late gene controls, respectively. The $\mathrm{BmN}$ cells transfected with lef-9KO-Bacmid, wt-Bacmid, and lef-9-Re-Bacmid DNA (1 $\mu \mathrm{g}$ each) were collected at $12,24,48$, and $72 \mathrm{hr}$. Total RNA was extracted using Trizol for qRT-PCR and treated with DNase I to remove residual BmNPV genomic DNA. Oligo dT ${ }_{(18)}$ was used as the primer for first-strand cDNA synthesis (Thermo Fisher Scientific, USA) by reverse transcription.

All primers were synthesized by the Shanghai Sangon Biotech Company (China) and PCR reagents were from TaKaRa Bio (Japan).

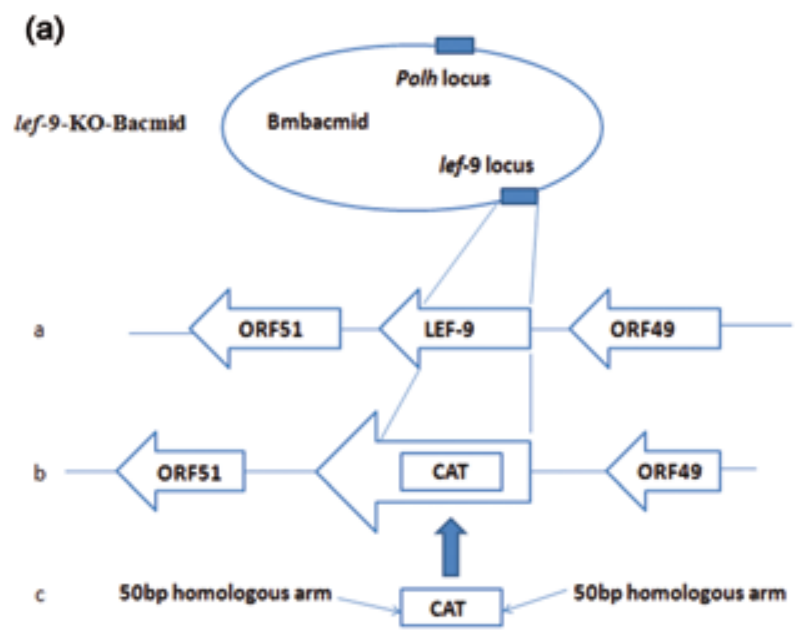

Virus titration assay. BmN cells were transfected with lef-9-KO-Bacmid, lef-9-Re-Bacmid, and wt-Bacmid (1 $\mu \mathrm{g}$ each), and then, the cell culture supernatants were carefully collected after 12, 18, 24, 48, 72, and $96 \mathrm{hr}$. At the logarithmic growth phase, $\mathrm{BmN}$ cells were transferred to 96 -well plates $\left(10^{3}\right.$ cells/well), and the virus supernatant was added at different time points, at $10 \times$ dilutions ranging from $10^{-1}$ to $10^{-9}$. Then, $10 \mu \mathrm{l}$ of viral suspension were added into each well, and 8-well repeats were used for each dilution gradient. Wells without any virus were used as negative controls. After incubation for 4 days, the cell incidence of each dilution was recorded, and the $50 \%$ tissue culture infective dose $\left(\mathrm{TCID}_{50}\right)$ was calculated using the Reed and Muench method.

Western blot assay. To analyze the effect of lef- 9 deficiency on the expression of viral genes, we studied the early protein Lef-3, the late protein VP39, and the very late protein $\mathrm{P} 10$ by using western blot analysis. The lef- $9-\mathrm{KO}$ virus, lef-9-repaired virus, and wild-type virus were used to transfect BmN cells, and cell lysates were collected after $72 \mathrm{hr}$ for sodium dodecyl sulfate polyacrylamide gel (SDSPAGE) electrophoresis and western blot analysis. Briefly, the cells were washed twice with PBS and lysed in lysis buffer (Beyotime Institute of Biotechnology, China) at $4^{\circ} \mathrm{C}$ and centrifuged at $12,000 \mathrm{rpm}$. The lysate $(10 \mu \mathrm{g}$ of protein per lane) was separated on $12 \%$ SDS-polyacrylamide gel, blotted onto nitrocellulose membrane and incubated with specific primary antibodies Lef-3, VP39, and P10 (1:1000) (Abmart Company, China). The expression of Lef -9 in cells transfected with the bacmids was analyzed by $6 \times$ His antibody (Zexiyuan Biotechnology Company, China). The antibodies were detected by a horseradish peroxidase-

(b)

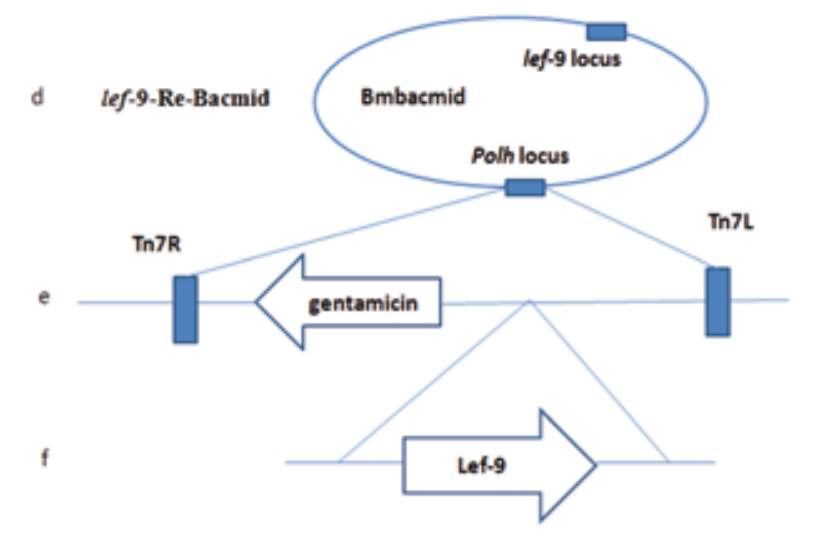

Fig. 1

Construction of lef-9 knockout (a) and lef-9-repaired (b) BmNPV bacmids

(a) Location of lef-9 in the lef-9-KO-Bacmid genome; (b) recombination between cat and lef-9; (c) PCR amplification of the target fragment using the pKD3 plasmid as a template and the primers lef-9-CF/lef-9-CR; (d) location of polh in the lef-9-Re-Bacmid genome; (e) insertion of lef-9 together with its promoter into the transposon; (f) PCR product of lef-9 and its promoter using the wild-type bacmid as a template. 
conjugated goat anti-mouse IgG antibody (DingGuo Biotechnology Company, China) using Western Bright ${ }^{\mathrm{TM}}$ ECL system (Advansta, USA).

Electron microscopy. BmN cells $\left(1 \times 10^{5} / 35-\mathrm{mm}\right.$ dish $)$ were transfected by $1 \mu \mathrm{g}$ each of lef-9-KO-Bacmid, wt-Bacmid, and lef-9-Re-Bacmid and collected after $24 \mathrm{hr}$. Cells were incubated overnight in $2.5 \%$ glutaraldehyde at $4^{\circ} \mathrm{C}$. Cell samples were prepared, and 70-90 nm sections were made using a microtome (Reichert, USA). The sections were treated with lead citrate solution and 50\% ethanol, and a saturated solution of uranyl acetate for $15 \mathrm{~min}$ for staining, and analyzed with a Hitachi H-7650 (Japan) transmission electron microscope. (a)

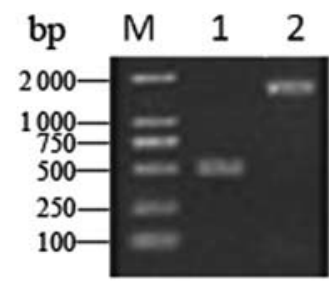

(b)

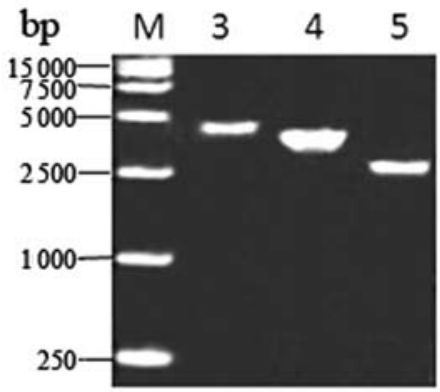

Fig. 2
Validation of $l e f-9$ knockout and repaired bacmids by PCR Lane M, DNA marker; lane 1, lef-9F and cat R PCR product; lane 2, lef-9F and lef-9R PCR product; lane 3, M13F and M13R PCR product; lane 4, M13F

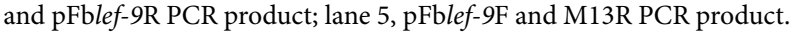

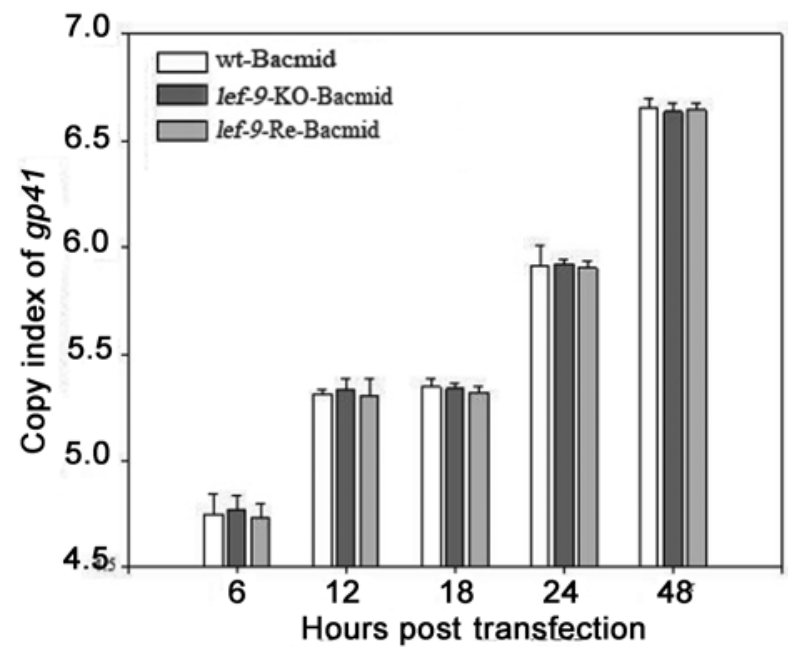

Fig. 3

Quantitative real-time PCR analysis of viral DNA replication Cells transfected with bacmids were harvested after 6-48 hr and assayed for gp41 by real-time PCR. Values are expressed as means \pm SEM.

\section{Results}

\section{Identification of lef-9-KO and lef-9-repaired bacmids}

Positive colonies proliferating on plates containing chloramphenicol and kanamycin were selected and confirmed by PCR, with extracted bacmid DNA as a template. The results showed that the PCR yielded 500 bp long product by using the lef-9F/cat $\mathrm{R}$ primers, and $1500 \mathrm{bp}$ long product when using the lef-9F/lef-9R primers (Fig. 2a). Size of the products was consistent with the theoretical sizes, which indicated that the lef-9-KO-Bacmid had been constructed successfully. The lef-9-repaired bacmid-positive colonies, were selected from plates containing kanamycin, gentamicin, tetracycline, IPTG, and X-gal, and confirmed by PCR. The primer combinations used were M13F/M13R, M13F/pFblef-9R, and pFblef-9F/ M13R (Table 1). PCR yielded a $4430 \mathrm{bp}$ long product with M13F/M13R primers, 3830 bp long product with M13F/ pFblef-9R primers, and $2690 \mathrm{bp}$ long product with $\mathrm{pFb}$ lef-9F/ M13R primers (Fig. 2b). The PCR products were consistent with the theoretical sizes, indicating that lef-9-Re-Bacmid had been successfully constructed.

\section{Viral replication analysis}

The total RNA extracted from the transfected cells collected at different time points was subjected to qRT-PCR. The Ct values related to $g p 41$ determined using qRT-PCR were converted to absolute copy numbers after calculations with a standard curve, with time after transfection on the abscissa and the copy number of the BmNPV-gp41 index on the or-

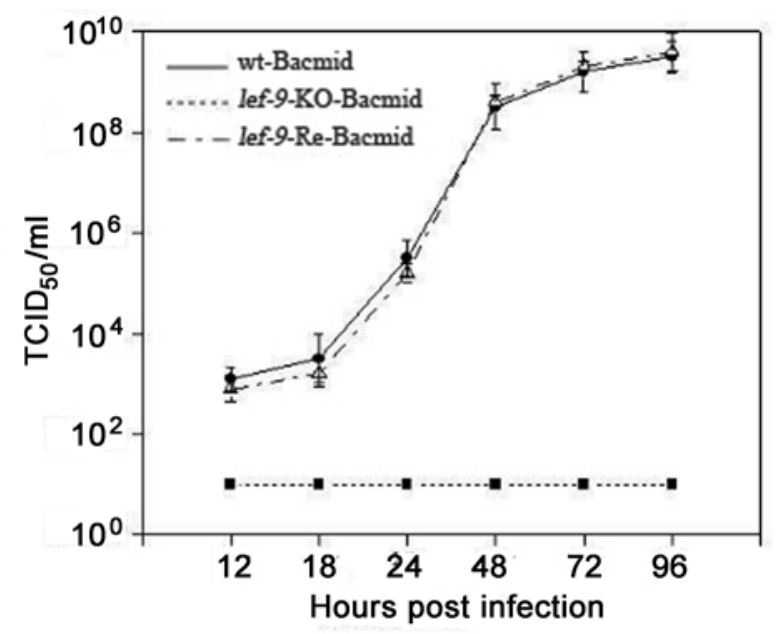

Fig. 4

Virus titers in BmN cells transfected with bacmids Cells transfected with the bacmids were harvested after 12-96 hr and assayed for virus titers. Values are expressed as means \pm SEM. 
dinate (Fig. 3). As shown in Fig. 3, there was no significant difference in viral DNA copy numbers $(P>0.05)$ between the silkworm BmN cells transfected with lef-9-KO-Bacmid, wtBacmid, and lef-9-Re-Bacmid, suggesting that the BmNPV lef-9 gene was not essential for genome replication.

The lef-9-KO-Bacmid had a TCID ${ }_{50}$ of 0 at all time points, indicating that the lef-9-KO virus did not produce contagious budding virus (BV; Fig. 4). The lef-9-KO-Bacmid lost the ability to produce infectious BV. In contrast, the wt-Bacmid and lef-9-Re-Bacmid retained this function, and generated increasing numbers of BVs with time after transfection. This result further confirms that lef- 9 is not indispensable for viral genome replication.

\section{Viral transcription analysis}

To study the effects of lef-9-KO bacmids on BmNPV gene transcription, we measured the transcription levels of the early genes lef-3 and $i e-1$, the late gene $v p 39$, and the very late gene p10. The qRT-PCR results showed that at $12 \mathrm{hr}$, the lef-3 transcription level in the lef-9-KO-Bacmid-transfected $\mathrm{BmN}$ cells showed little difference from the levels in the wt-Bacmid- and (a)

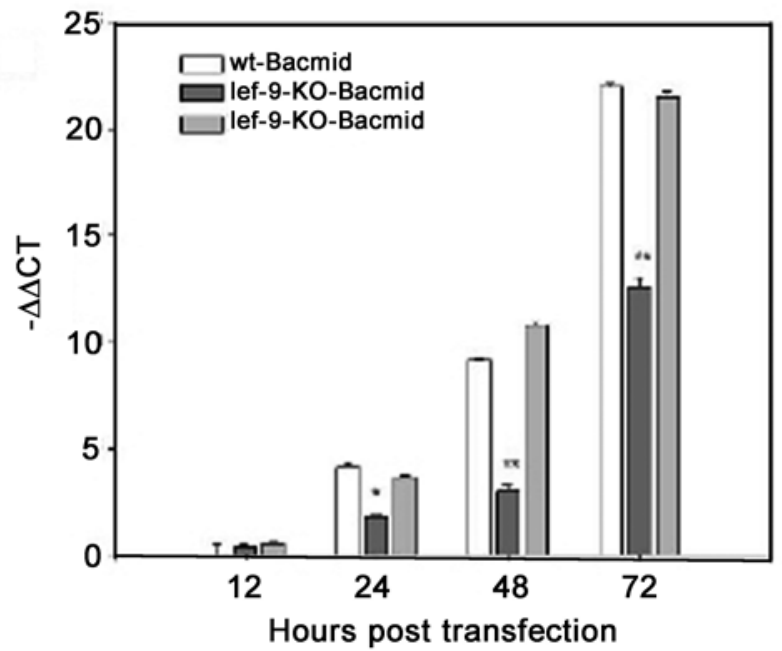

(c)

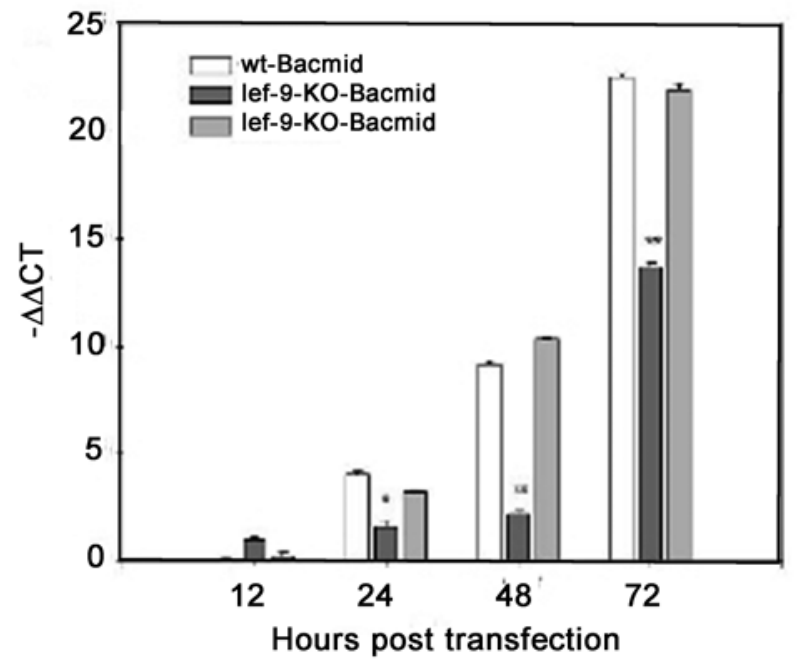

(b)

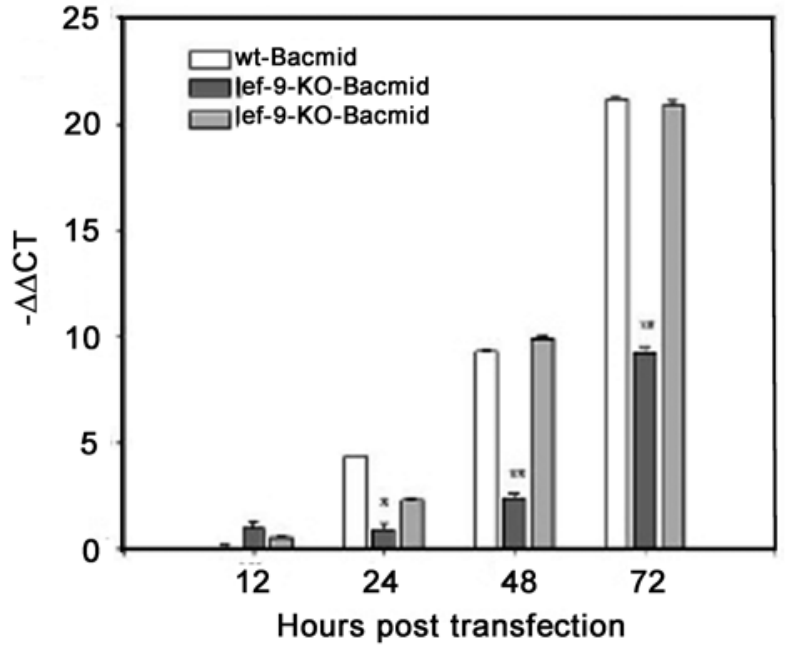

(d)

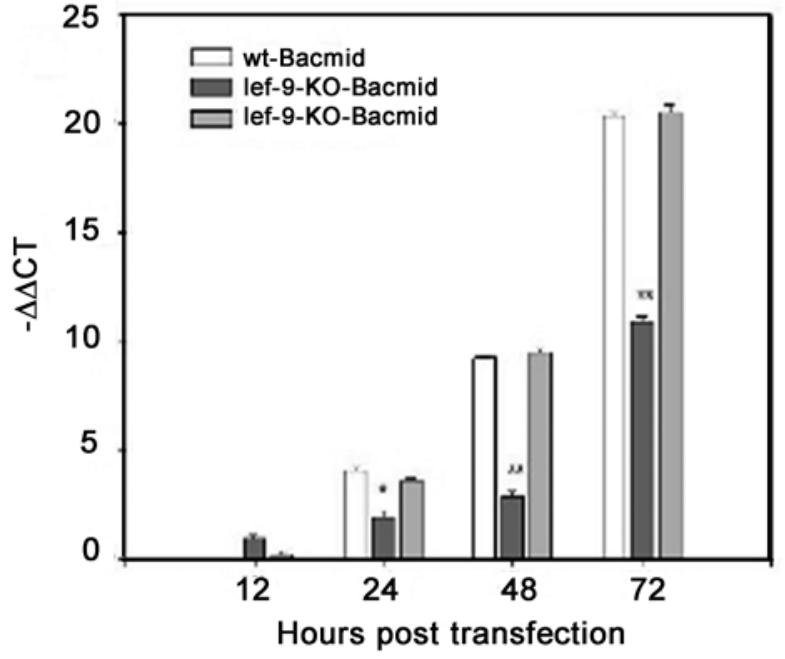

Fig. 5

Transcrition levels of $l e f-3(\mathrm{a}), i e-1$ (b), $v p 39$ (c) and $p 10$ (d) in cells transfected with bacmids Transcription levels were analyzed by real-time PCR. ${ }^{\star} \mathrm{P}<0.05,{ }^{* *} \mathrm{P}<0.01$ vs. wt-Bacmid or lef-9-Re-Bacmid at $12,24,48$, and $72 \mathrm{hr}$. Values are expressed as means \pm SEM. Similar results were obtained in three independent experiments. 
lef-9-Re-Bacmid-transfected cells (Fig. 5a). At $24 \mathrm{hr}$ however, lef-3 expression was significantly lower in the lef-9-KO cells than in the wt-Bacmid- and lef-9-Re-Bacmid-transfected cells $(P<0.05)$. Furthermore, these significant differences retained at 48 and $72 \mathrm{hr}(P<0.01)$. Analysis of the early gene ie-1 (Fig. 5b), the late gene $v p 39$ (Fig. $5 c$ ), and the very late gene $p 10$ (Fig. 5d) showed similar trends to those observed with lef-3. Therefore, we speculated that the lef-9 gene was positively correlated with virus genome transcription, including the transcription of early, late, and very late genes. The deficiency of the lef-9 gene had a great influence on the viral genome transcription level.

\section{Western blot analysis}

The western blot (Fig. 6a) results showed that the lysates of BmN cells transfected with lef-9-Re-Bacmids had obvi- ous bands, which indicated that the lef-9-Re-Bacmid had been successfully constructed and that Lef- 9 was correctly expressed. No obvious or very low protein expression of Lef-3, VP39, and P10 was detected in the lysates of BmN cells transfected with lef-9-KO-Bacmid DNA when compared with cells transfected with lef-9-Re-Bacmid, indicating that lef- 9 was essential for the expression of early and late viral genes (Fig. 6b).

\section{Viral assembly analysis}

To analyze the effect of lef- 9 deficiency on virion assembly, we examined transfected $\mathrm{BmN}$ cells under a transmission electron microscope. As shown in Fig. 7, no enveloped baculovirus was observed in the $\mathrm{BmN}$ cells transfected with lef-9-KO bacmids (Fig. 7 d-f). However, BmN cells
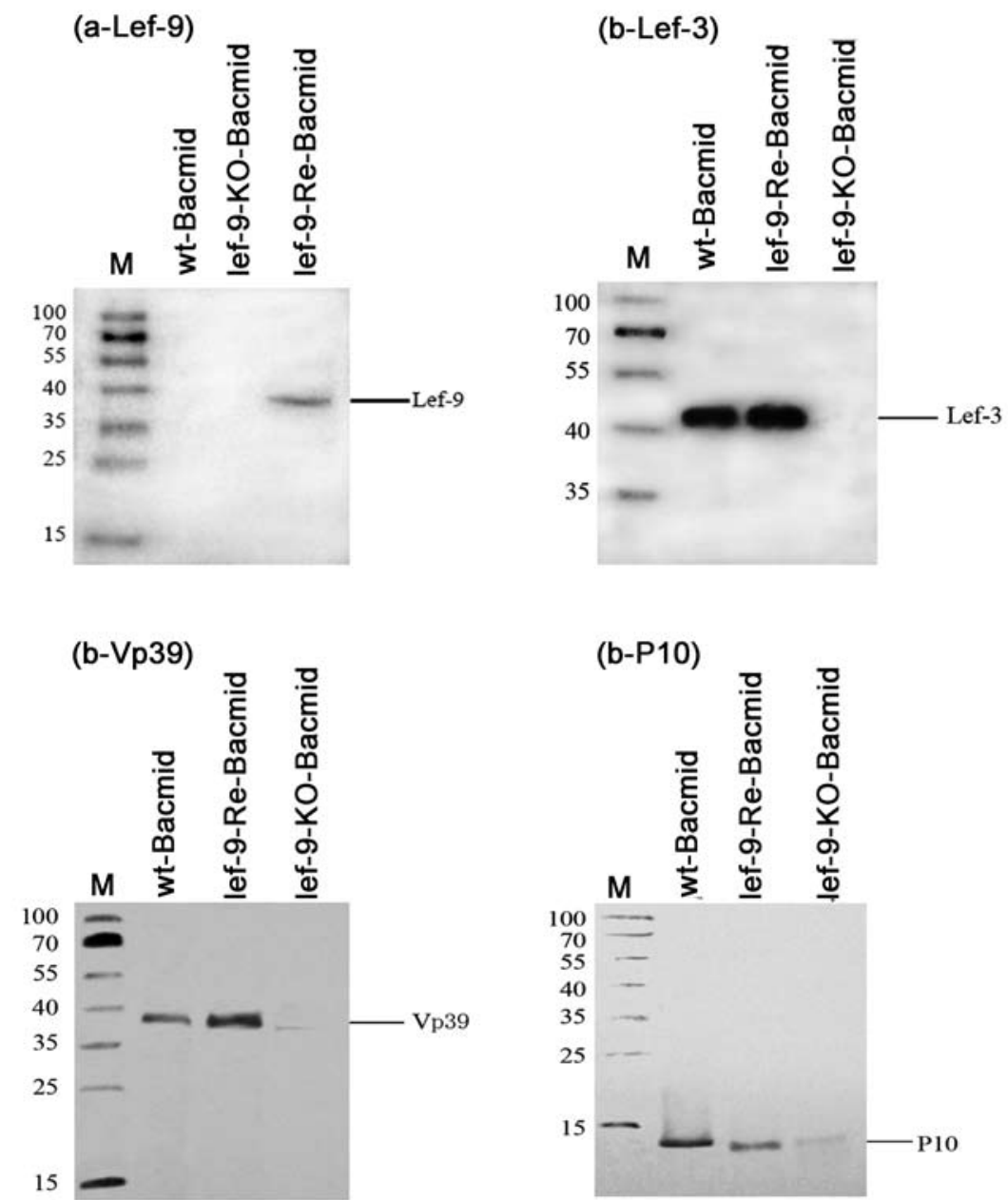

Fig. 6

Western blot analysis of Lef-9 (a) and Lef-3, VP39 and P10 (b)

The expression of Lef- 9 was detected by $6 \times$ His monoclonal antibody. The expressions of Lef- 3 , P10, and VP39 were detected by Lef-3, VP39, and P10 monoclonal antibodies, respectively. The lysates of BmN cells transfected with wt-Bacmid DNA (wt-Bacmid), lef-9-KO-Bacmid DNA (lef-9-KO-Bacmid) and lef-9-Re-Bacmid DNA (lef-9-Re-Bacmid) were used. Lane M, protein marker. 
(a)

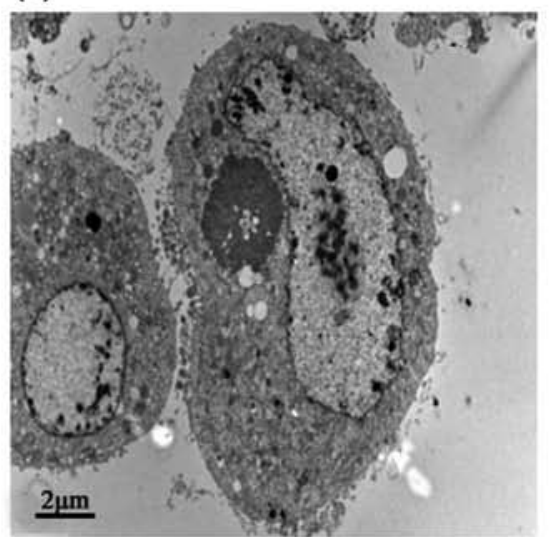

(d)

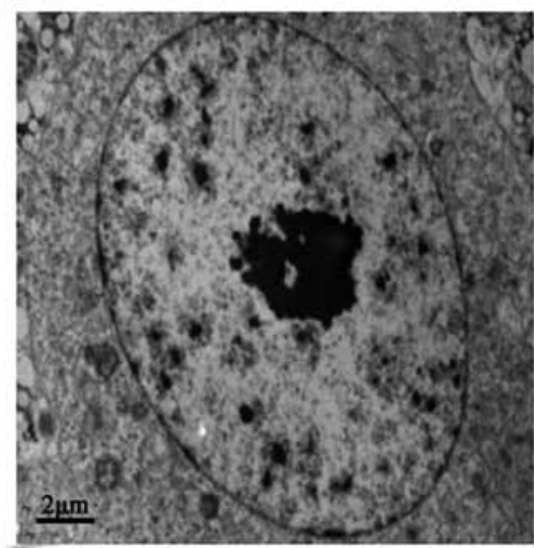

(g)

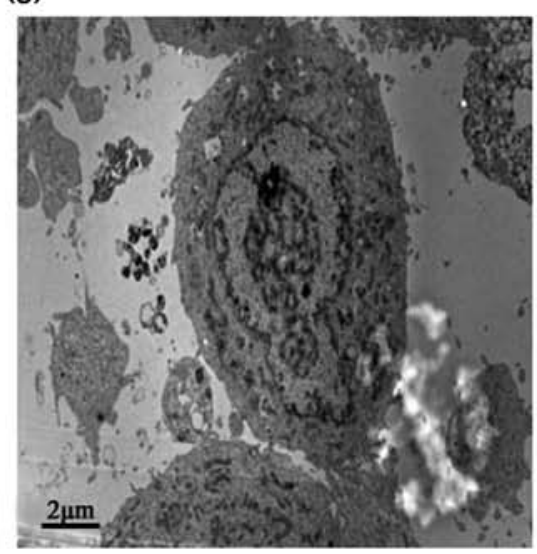

(b)

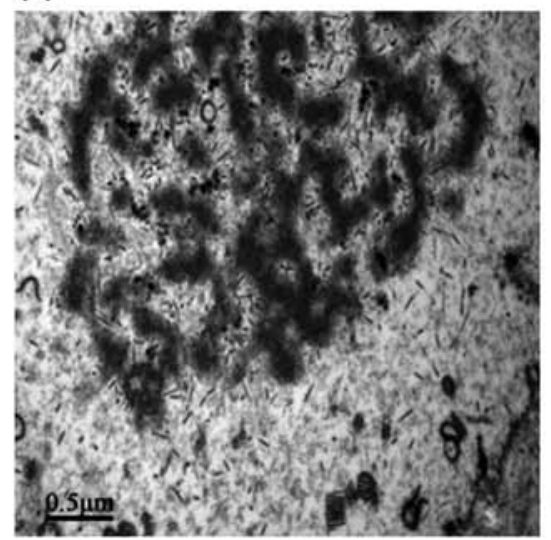

(e)

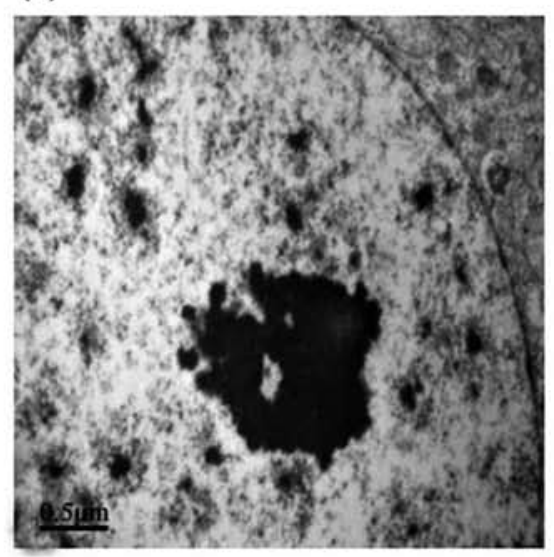

(h)

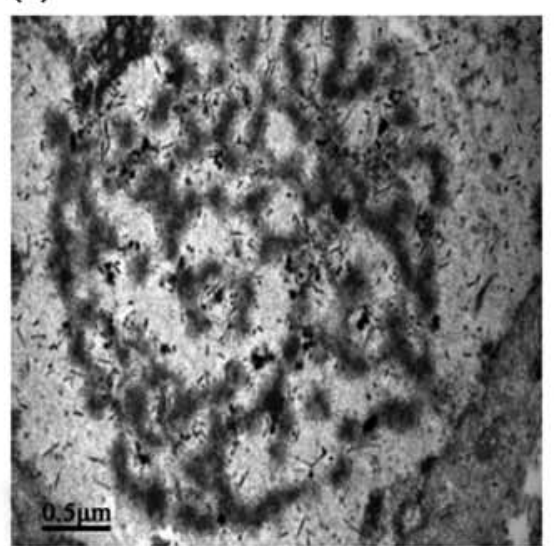

(c)

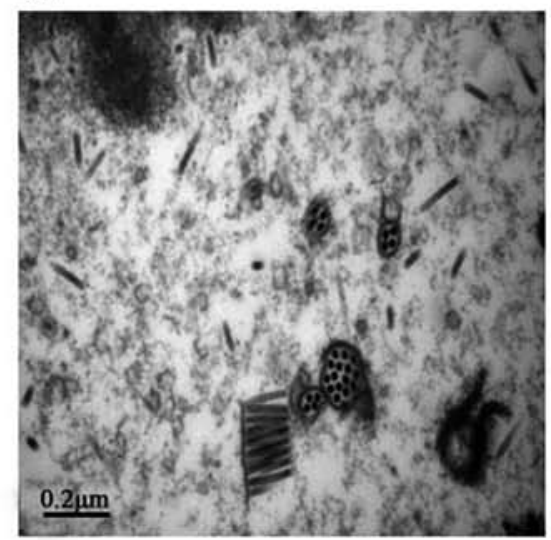

(f)

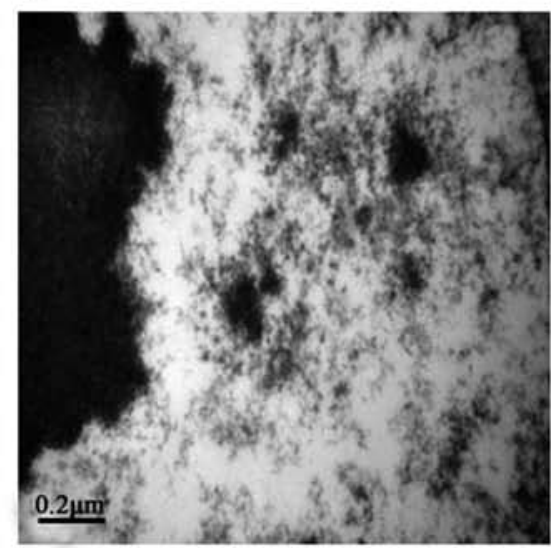

(i)

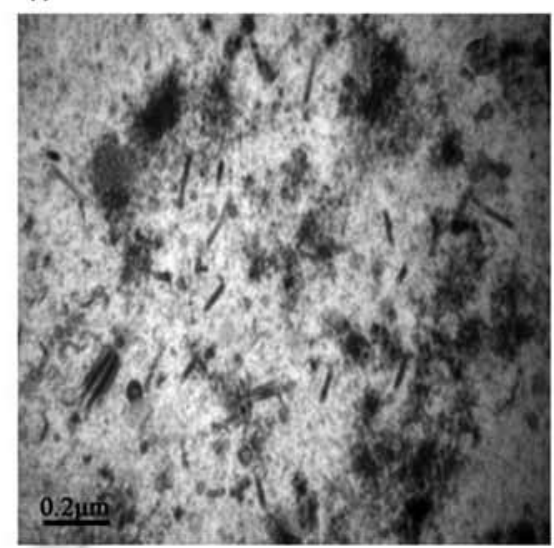

Fig. 7

Electron microscopic analysis of BmN cells transfected with wt-Bacmid, lef-9-KO-Bacmid, and lef-9-Re-Bacmid

A-C, $24 \mathrm{hr}$ after transfection with wt-Bacmid (A, 5,000x; B, 20,000x; C, 50,000x); D-F, $24 \mathrm{hr}$ after transfection with lef-9-KO-Bacmid (D, 5,000x; E, 20,000×; F, 50,000×); G-I, 24 hr after transfection with lef-9-Re-Bacmid (G, 5,000×; H, 20,000×; I, 50,000×).

transfected with wild-type virus (Fig. 7 a-c) or lef-9-repaired virus (Fig. $7 \mathrm{~g}$-i) showed typical characteristics, including an electron-dense virogenic stroma enriched with rod-shaped enveloped nucleocapsids formed in the nucleus (Fig. 7c).

\section{Discussion}

In this study, we constructed the lef-9-KO-Bacmid using the Red recombination technique and the Bac-to-Bac 
baculovirus expression system to construct a lef-9-repaired virus. The deficient virus DNA was used to transfect $\mathrm{BmN}$ cells. We used $g p 41$ as a reference for determining the viral copy index. The $g p 41$ gene, encoding the GP41 protein, is essential for viral replication, and is required for the egress of nucleocapsids from the nucleus during BV synthesis (Zhang et al., 2014). Defective viral titers in cell supernatants were 0 at all time points after transfection (Chikako et al., 2012), showing that the lef-9-KO virus had lost its vitality and ability to proliferate; in contrast, the lef-9-repaired virus regained its infectivity. Studies have shown that many factors can prevent viruses from forming infectious BVs, especially, factors related to viral DNA replication (Milks et al., 2003). However, our results showed that the intracellular viral genome copy number did not significantly differ between the lef-9-KOBacmid, wt-Bacmid, and lef-9-Re-Bacmid. Therefore, we concluded that the lef-9 gene was not essential for BmNPV genome replication, but was vital for viral gene transcription, which explains the failure to create infectious BVs. The knockout of the late expression factors lef-10 (Yu et al., 2013) and vlf-1 (Vanarsdall et al., 2004) can reduce viral DNA copies, affect early and late gene transcription, and prevent BV production and spread. In addition, lef-9, a BmNPV late expression factor, has been shown to be important for viral gene transcription (Acharya and Gopinathan, 2002). Our results pertaining to the lef-9-KO virus were similar to these findings, for instance, the inability to produce infectious BVs (Vanarsdall et al., 2004), although we found that early viral genome replication was not affected.

To further study the biological function of the lef- 9 gene, we examined the effects of a lef-9-KO virus on the transcription and expression of viral genes. The results showed that lef-9 deficiency affected the transcription and expression of early, late, and very late viral genes. Lef-9 is a subunit of viral RNA polymerase (Guarino et al., 1998; Vanarsdall et al., 2005), so it is very reasonable that lef-9 deficiency will affect the transcriptional levels of viral early and late genes, leading to the failure of the expression of these proteins. Western blots showed that lef-9-KO-Bacmid-transfected $\mathrm{BmN}$ cells had no obvious detectable expression of Lef-3, VP39, and P10 proteins. These results further confirmed that the deletion of the lef- 9 gene greatly reduced the transcription levels of early, late, and very late genes.

Finally, we used transmission electron microscopy to observe BmN cells transfected with wt-Bacmid, lef-9-KOBacmid, and lef-9-Re-Bacmid. In the lef-9-KO virus-infected cells, we did not observe any mature virions or the formation of a virus particle sac surrounded by a membrane. This result confirmed that the loss of lef- 9 affected virus assembly, and therefore, no virus particles with infective activity could be produced. BmN cells infected with the lef-9-repaired virus or wild-type virus showed typical symptoms of baculovirus infection, including a highly electron-dense virogenic stroma, enlarged nuclei, and abundant nucleocapsid assembly in the matrix. These results were consistent with the results of virus titer measurements in cells transfected with the bacmids.

In summary, lef-9 is necessary for the production of BVs in cells. The absence of this gene will affect viral gene transcription and expression, and prevent normal virus assembly.

Acknowledgements. This work was supported by the National High-tech R\&D program (863 Program) (No. 2011AA100603) and the Project Grants 521 Talents Cultivation of Zhejiang Sci-Tech University (No. 11610132521506).

\section{References}

Acharya A, Gopinathan KP (2002): Characterization of late gene expression factors lef- 9 and lef- 8 from Bombyx mori nucleopolyhedrovirus. J. Gen. Virol. 83, 2015-2023. http:// dx.doi.org/10.1099/0022-1317-83-8-2015

Ayres MD, Howard SC, Kuzio J, Lopez-Ferber M, Possee RD (1994): The complete DNA sequence of Autographa californica nuclear polyhedrosis virus. Virology 202, 586-605. http:// dx.doi.org/10.1006/viro.1994.1380

Bulach DM, Kumar CA, Zaia A, Liang B, Tribe DE (1999): Group II nucleo-polyhedrovirus subgroups revealed by phylogenetic analysis of polyhedrin an-d DNA polymerase gene sequences. J. Invertebr. Pathol. 73, 59-73. http://dx.doi. org/10.1006/jipa.1998.4797

Chikako O, Takanori K, Hitomi T, Ken S, Shinichiro A, Hisanori B (2012): Phenotypic grouping of $141 \mathrm{BmNPVs}$ lacking viral gene sequences. Virus Res. 165, 197-206. http:// dx.doi.org/10.1016/j.virusres.2012.02.016

Crouch EA, Cox LT, Morales KG, Passarelli AL (2007): Inter-subunit interactions of the Autographa californica M nucleopolyhedrovirus RNA polymerase. Virology 367, 265-274. http://dx.doi.org/10.1016/j.virol.2007.05.026

Copeland NG, Jenkins NA, Court DL (2001): Recombineering: a powerful new tool for mouse functional genomics. Nat. Rev. Genet. 2, 769-779. http://dx.doi. org/10.1038/35093556

Costantino N, Court DL (2003): Enhanced levels of lambda Redmediated recombinants in mismatch repair mutants. Proc. Natl. Acad. Sci .USA 100, 15748-15753. http:// dx.doi.org/10.1073/pnas.2434959100

Gomi S, Majima K, Maeda S (1999): Sequence analysis of the genome of Bombyx mori nucleopolyhedrovirus. J. Gen. Viro. 180, $1323-$ 1337. http://dx.doi.org/10.1099/0022-1317-80-5-1323

Guarino LA, Xu B, Jin J, Dong W (1998): A virus-encoded RNA polymerase purified from baculovirus-infected cells. J. Virol. 72, 7985-7991.

Hang X, Dong W, Guarino LA (1995): The lef-3 gene of Autographa californica nuclear polyhedrosis virus encodes a singlestranded DNA-binding protein. J. Virol. 69, 3924-3928.

Herniou EA, Olszewski JA, Cory JS, O'Reilly DR (2003): The genome sequence and evolution of baculoviruses. Annu. Rev. Entomol. 48, 211-234. http://dx.doi.org/10.1146/ annurev.ento.48.091801.112756 
Herniou EA, Jehle JA (2007): Baculovirus phylogeny and evolution. Curr. Drug Targets 8, 1043-1050. http://dx.doi. org/10.2174/138945007782151306

Iyengar B, Roote J, Campos AR (1999): The tamas gene, identified as a mutation that disrupts larval behavior in Drosophila melanogaster, codes for the mitochondrial DNA polymerase catalytic subunit (DNApol-gamma125). Genetics $153,1809-1824$.

Jehle JA, Blissard GW, Bonning BC, Cory JS, Herniou EA, Rohrmann GF, T heilmann DA, Thiem SM, Vlak JM (2006): On the classification and nomenclature of baculoviruses: a proposal for revision. Arch. Virol. 151, 1257-1266. http://dx.doi.org/10.1007/s00705-006-0763-6

Kejju O, Victor SM, Susumu M (1999): Colocalization of baculovirus IE- 1 and two DNA-binding proteins, DBP and LEF-3, to viral replication factories. J. Virol. 73, 110-119.

Lin G, Blissard GW (2002): Analysis of an Autographa californica nucleopoly-hedrovirus lef-11 knockout: LEF-11 is essential for viral DNA replication. J. Virol. 76, 2770-2779. http://dx.doi.org/10.1128/JVI.76.6.2770-2779.2002

Lu A, Miller LK (1995): The roles of eighteen baculovirus late expression factor genes in transcription and DNA replication. J. Virol. 69, 975-982.

Milks ML, Washburn JO, Willis LG, Volkman LE, Theilmann DA (2003): Deletion of pe38 attenuates AcMNPV genome replication, budded virus production, and virulence in heliothis virescens. Virology 310, 224-234. http://dx.doi. org/10.1016/S0042-6822(03)00143-0

Rapp JC, Wilson JA, Miller LK (1998): Nineteen baculovirus open reading frames, including LEF-12, support late gene expression. J. Virol. 72, 10197-10206.

Rose J, Kleespies RG, Wang Y, Wennmann JT, Jehle JA (2013): On the susceptibility of the box tree moth Cydalima perspectalis to Anagrapha falcifera nucleopolyhedrovirus (AnfaNPV). J. Invertebr. Pathol. 113, 191-197. http:// dx.doi.org/10.1016/j.jip.2013.03.009

Shi Y, Zhang C, Xie C, Quan Y, Nie Z, Chen J, Lv Z, Zhang Y, Yu $\mathrm{W}$ (2015): The effect of BM67 gene deletion on Bombyx mori nuclear polyhedrosis virus replication. Acta Virol. 59, 40-48. http://dx.doi.org/10.4149/av 2015 $01 \quad 40$

Su J, Lung O, Blissard GW (2011): The Autographa californica multiple nucleopolyhedrovirus lef- 5 gene is required for productive infection. Virology 416, 54-64. http://dx.doi. org/10.1016/j.virol.2011.04.019

Vanarsdall AL, Okano K, Rohrmann GF (2004): Characterization of a baculovirus with a deletion of vlf-1. Virology 326, 191-201. http://dx.doi.org/10.1016/j.virol.2004.06.003

Vanarsdall AL, Okano K, Rohrmann GF (2005): Characterization of the replication of a baculovirus mutant lacking the DNA polymerase gene. Virology 331, 175-180. http://dx.doi. org/10.1016/j.virol.2004.10.024

Vanarsdall AL, Okano K, Rohrmann GF (2006): Characterization of the role of very late expression factor 1 in baculovirus capsid structure and DNA processing. J. Virol. 80, 1724-1733. http://dx.doi.org/10.1128/JVI.80.4.1724-1733.2006

Wu Y, Wu Y, Wu Y, Tang H, Wu H, Zhang G, Wang W (2013): Screening of candidate proteins interacting with IE-2 of Bombyx mori nucleopolyhedrovirus. Mol. Biol. Rep. 40, 5797-804. http://dx.doi.org/10.1007/s11033-013$\underline{2683-3}$

Yin F, Zhu Z, Liu X, Hou D, Wang J, Zhang L, Wang M, Kou Z, Wang H, Deng F, Hu Z (2015): The complete genome of a new Betabaculovirus from Clostera anastomosis. PLoS One. 10, e0132792. http://dx.doi.org/10.1371/journal. pone. 0132792

Yu W, Du CY, Quan YP, Nie ZM, Chen J, Lv ZB, Zhang YZ (2013): Characterization of late gene expression factor LEF-10 from Bombyx mori nucleopolyhedrovirus. Virus Res. 175, 45-51. http://dx.doi.org/10.1016/j. virusres.2013.03.022

Zhang J, He Q, Zhang CD, Chen XY, Chen XM, Dong ZQ, Li N, Kuang XX, Cao MY, Lu C, Pan MH (2014): Inhibition of BmNPV replication in silk-worm cells using inducible and regulated artificial microRNA precursors targeting the essential viral gene lef-11. Antiviral Res. 104, 143-152. http://dx.doi.org/10.1016/j.antiviral.2014.01.017 